\title{
LA-UR- $00-3612$
}

Approved for public release distribution is unlimited.

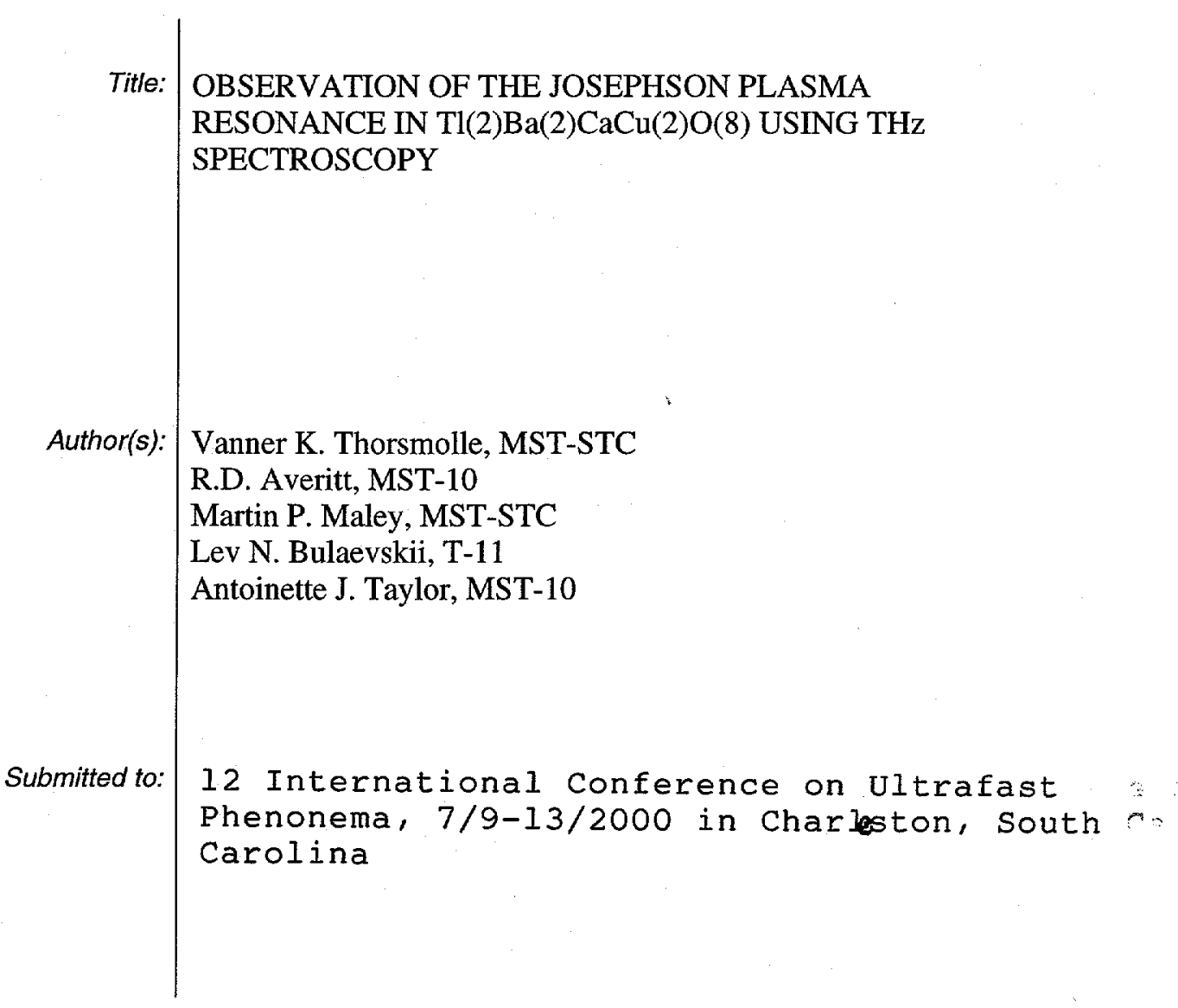

\section{Los Alamos NATIONAL LABORATORY}

Los Alamos National Laboratory, an affirmative action/equal opportunity employer, is operated by the University of California for the U.S. Department of Energy under contract W-7405-ENG-36. By acceptance of this article, the publisher recognizes that the U.S. Government retains a nonexclusive, royalty-free license to publish or reproduce the published form of this contribution, or to allow others to do so, for U.S. Government purposes. Los Alamos National Laboratory requests that the publisher identify this article as work performed under the auspices of the U.S. Department of Energy. Los Alamos National Laboratory strongly supports academic freedom and a researcher's right to publish; as an institution, however, the Laboratory does not endorse the viewpoint of a publication or guarantee its technical correctness. 


\section{DISCLAIMER}

This report was prepared as an account of work sponsored by an agency of the United States Government. Neither the United States Government nor any agency thereof, nor any of their employees, make any warranty, express or implied, or assumes any legal liability or responsibility for the accuracy, completeness, or usefulness of any information, apparatus, product, or process disclosed, or represents that its use would not infringe privately owned rights. Reference herein to any specific commercial product, process, or service by trade name, trademark, manufacturer, or otherwise does not necessarily constitute or imply its endorsement, recommendation, or favoring by the United States Government or any agency thereof. The views and opinions of authors expressed herein do not necessarily state or reflect those of the United States Government or any agency thereof. 


\section{DISCLAIMER}

Portions of this document may be illegible in electronic image products. Images are produced from the best available original document. 


\title{
Observation of the Josephson Plasma Resonance in $\mathrm{Tl}_{2} \mathrm{Ba}_{2} \mathrm{CaCu}_{2} \mathrm{O}_{8}$ using $\mathrm{THz}$ Spectroscopy
}

\author{
V. K. Thorsmølle, R. D. Averitt, M. P. Maley, L. N. Bulaevskii and \\ A. J. Taylor \\ Los Alamos National Lab, Los Alamos, NM 87545 \\ E-mail: vthorsmolle@lanl.gov, ttaylor@lanl.gov
}

\begin{abstract}
Employing terahertz time-domain spectroscopy in transmission, we have unambiguously observed the Josephson Plasma Resonance in $\mathrm{Tl}_{2} \mathrm{Ba}_{2} \mathrm{CaCu}_{2} \mathrm{O}_{8}$ high- $\mathrm{T}_{\mathfrak{c}}$ thin films in zero magnetic field as a function of temperature.
\end{abstract}

\section{Introduction}

The c-axis Josephson Plasma Resonance (JPR) in highly anisotropic layered cuprate superconductors originates from the interlayer tunneling of Cooper pairs. These Josephson-coupled systems have received much interest in recent years due to their electrodynamic properties. The JPR, related to the c-axis penetration length, $\omega_{p c}=c / \lambda_{c}\left(\varepsilon_{\infty}^{c}\right)^{1 / 2}$, is a fundamental probe of the superconducting state and an excellent tool to study these highly anisotropic systems. For $\mathrm{T} \ll \mathrm{T}_{c}$, the spectral shift of the JPR with temperature is related to the symmetry of the order parameter. For $T$ close to $T_{c}$, the appearance of the JPR probes the onset of interlayer phase coherence. Furthermore, the JPR spectral width is a measure of the quasiparticle scattering rate. In addition, the JPR probes the tunneling mechanism and provides information about the validity of the interlayer-tunneling model [1]. In a magnetic field, the JPR probes the correlation of pancake vortices along the c-axis and is a tool to study the B-T phase diagram [2,3]. The advantage of using terahertz time-domain spectroscopy (TTDS) to map out the phase diagram is that this technique allows measurements to be made over a broad frequency range with fixed magnetic fields and temperatures. Importantly, the JPR and the quasiparticle scattering rates of high- $\mathrm{T}_{c}$ superconductors with extreme anisotropy such as the BSCCO, thallium, or mercury based compounds lie in the $\mathrm{THz}$ frequency range.

We have measured the JPR in $\mathrm{Tl}_{2} \mathrm{Ba}_{2} \mathrm{CaCu}_{2} \mathrm{O}_{8}$ (T1-2212) thin films in zero applied field as a function of temperature employing terahertz spectroscopy in transmission. The JPR frequency peak is $692 \mathrm{GHz}$ at $10 \mathrm{~K}$ decreasing to $386 \mathrm{GHz}$ at $90 \mathrm{~K}\left(\mathrm{~T}_{\mathrm{c}}=98.8 \mathrm{~K}\right)$. We also observe an increase in the JPR spectral width with increasing temperature, indicative of an increase in the quasiparticle scattering rate.

Page 1 Thorsmolle, et al. 


\section{Results and Discussion}

The experiments were carried out in transmission using TTDS. The experimental setup is shown and explained in [4]. The Tl-2212 film ( $-700 \mathrm{~nm}$ thick) was grown on an MgO substrate using a two-step process. First an amorphous TBCCO film was deposited by laser ablation. Next it was annealed at high temperature in oxygen to form the 2212 phase. The measured $T_{c}$ for this film was $98.8 \mathrm{~K}$.

In order to excite the c-axis JPR, a component of the terahertz field has to be along the c-axis of the sample. The a-b plane plasma frequency is on the order of $1.5 \mathrm{eV}$, while the JPR along the c-axis lies in the terahertz range. Therefore, tilting the sample at an angle $\Theta$ will transmit the incident p-polarized electric field component parallel to the c-axis at frequencies above the plasma edge while reflecting the component that is parallel to the $a-b$ plane. A more detailed analysis shows that for frequencies $\omega<\omega_{a b}$, and taking into account that the system consists of discrete layers, the electromagnetic waves can only propagate along the c-axis in a narrow frequency window above the plasma edge.
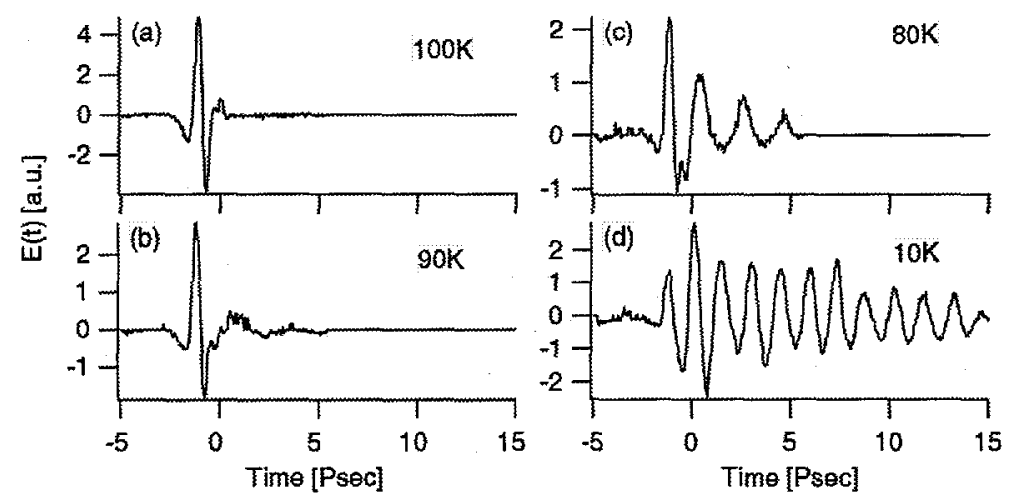

Fig 1. (a)-(d)) Transmission of incident THz pulse through Tl-2212 thin film. (a) The THz pulse transmitted through the film above the onset of superconductivity. (b) Just below $\mathrm{T}_{c}$, there is a slight oscillation after the main pulse due to excitation of the c-axis JPR - the oscillation is highly damped. (c) At $80 \mathrm{~K}$, the JPR oscillation is more pronounced since the quasiparticle density is reduced resulting in a decrease in the scattering. (d) At $10 \mathrm{~K}$, the JPR oscillations persist at least to $15 \mathrm{ps}$ - note also the increased oscillation frequency in comparison to (c).

Figure 1(a)-(d) shows the transmission of a THz pulse through a Tl-2212 thin film as a function of temperature. Fig. 1(a) shows the $\mathrm{THz}$ pulse transmitted through the film above the onset of superconductivity. At 90K (Fig. 1(b)), the onset of caxis coherent Josephson tunneling is just perceptible as a slight oscillation after the main THz pulse. The JPR is highly damped due to quasiparticle scattering. At lower temperatures (Fig. 1(c)-(d)), the JPR is more pronounced due to a reduction in

Page 2 Thorsmolle, et al. 
scattering. The time domain data also indicates that the JPR shifts to higher frequencies as the temperature is lowered as indicated by the increase in oscillation frequency.
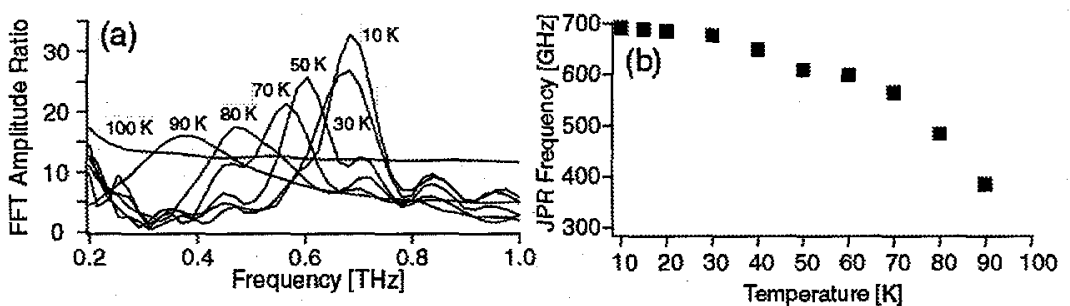

Fig 2. (a) The JPR as a function of frequency at various temperatures. With increasing temperature, the peak shifts to lower frequencies and broadens. (b) The JPR peak maximum as a function of temperature.

Figure 2 shows the amplitude ratio of the Fast Fourier Transform (FFT) of the time-domain data in Fig. 1 divided by a reference scan on bare MgO. Fig. 2(a) clearly shows that the JPR shifts to higher frequencies and narrows as the temperature is decreased. The JPR frequency shift vs. temperature in Fig. 2(b) agrees well with previous reflectivity measurements on Tl-2212 [1] and we have been able to extend the measurements to temperatures approaching $T_{c}$. Future experiments aim to utilize this new capability of measuring the JPR in transmission to probe the $\mathrm{B}-\mathrm{T}$ vortex phase diagram of anisotropic high- $\mathrm{T}_{\mathrm{c}}$ superconductors.

Acknowledgements. We would like to thank Superconductor Technologies Inc. for providing the $\mathrm{Tl}-2212$ films used in these experiments. This work was funded in part by CLC.

\section{References}

A.A. Tsvetkov, et al., Nature 395, 360 (1998).

L.N. Bulaevskii, M.P. Maley, M. Tachiki, Phys. Rev. Lett. 268, 809 (1995).

A.E. Koshelev, Phys. Rev. Lett. 77, 3901 (1996).

R.D. Averitt, G. Rodriguez, J.L.W. Siders, S.A. Trugman, A.J. Taylor, J. Opt. Soc. Am. B. 17, 327 (2000).

Page 3 Thorsmolle, et al. 